\begin{tabular}{|c|c|c|c|c|c|c|}
\hline \multirow{4}{*}{ Impact Factor: } & ISRA (India) & $=3.117$ & SIS (USA) & $=0.912$ & ICV (Poland) & $=6.630$ \\
\hline & ISI (Dubai, UAE & $=0.829$ & РИНЦ (Russia) & $=0.156$ & PIF (India) & $=1.940$ \\
\hline & GIF (Australia) & $=0.564$ & ESJI (KZ) & $=8.716$ & IBI (India) & $=4.260$ \\
\hline & JIF & $=1.500$ & SJIF (Morocco) & $=5.667$ & OAJI (USA) & $=0.350$ \\
\hline
\end{tabular}

SOI: $1.1 /$ TAS $\quad$ DOI: $10.15863 / T A S$
International Scientific Journal
Theoretical \& Applied Science
p-ISSN: $2308-4944$ (print) $\quad$ e-ISSN: $2409-0085$ (online)
Year: $2019 \quad$ Issue: $05 \quad$ Volume: 73
Published: $25.05 .2019 \quad$ http://T-Science.org

SECTION 25. Technologies of materials for the light and textile industry
QR - Issue

QR - Article
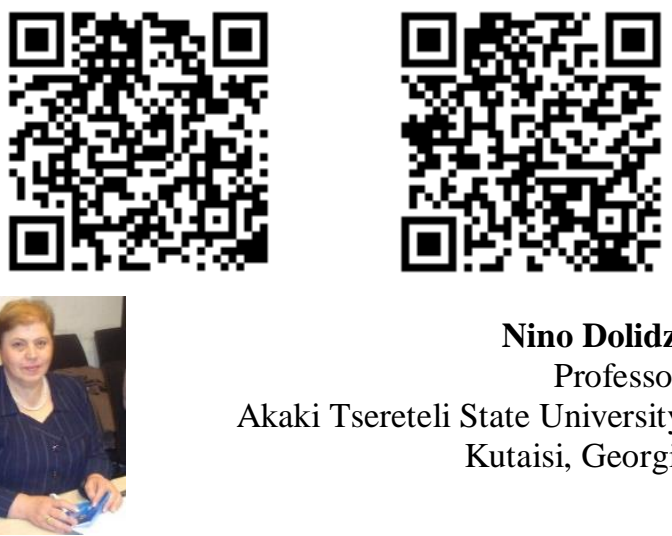

Nino Dolidze

Professor,

Akaki Tsereteli State University,

Kutaisi, Georgia

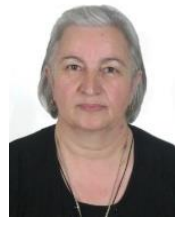

Qetevan Chirgadze

Academic doctor,

Akaki Tsereteli State University,

Kutaisi, Georgia

Lia Lursmanashvili

Professor,

Georgian Technical University,

Tbilisi, Georgia

\title{
THE RELEVANCE OF THE STUDY OF VIOLATIONS OF POSTURE OF CHILDREN'S POPULATION OF GEORGIA
}

Abstract: The purpose of the study was the detection of violations of posture of children's population of Georgia. To match our purpose an anthropomorphological study of children of primary and secondary school age and adolescents was conducted. Our study revealed that the violations of posture are more frequent in children at age of 6 to 13 years. Timely detection of the problem is of great importance for the prevention of further complications and scoliosis, today's most common disease in the world. As a result of the study the cases of violations of posture were determined and their quantitative characteristics were identified. Received data suggests the need for a mass anthropomorphological study of the children's population of Georgia. The results of the study will be used to design corrective means in order to eliminate violations of posture.

Key words: posture, anthropomorphological study, chuldren's population, scoliosis.

Language: Russian

Citation: Dolidze, N., Chirgadze, Q., \& Lursmanashvili, L. (2019). The relevance of the study of violations of posture of children's population of Georgia. ISJ Theoretical \& Applied Science, 05 (73), 286-291.

Soi: http://s-o-i.org/1.1/TAS-05-73-41 Doi: crossef https://dx.doi.org/10.15863/TAS.2019.05.73.41

АКТУАЛЬНОСТЬ ИССЛЕДОВАНИЯ НАРУШЕНИЙ ОСАНКИ ДЕТСКОГО НАСЕЛЕНИЯ ГРУЗИИ

Аннотация: Целью нашего исследование было выявление случаев нарушения осанки детского населения Грузии. Для решения поставленных задач проводилось антропоморфологическое исследование детей младшего и старшего школьного возраста и подростков. Исследование показало, что нарушение осанки наиболее выражено у детей младшего школьного возраста от 6 до 13 лет. Соответственно, своевременное выявление проблемы имеет большое значение для предотвращение дальнейших осложнений и профилактики сколиоза, на сегодняшний день самого распространенного заболевания в мире. В результате исследования были выявлены случаи нарушения осанки детей и определены их количественные показатели. Полученные данные указывают на необходимость проведения массового антропоморфологического исследования детского населения Грузии. Итоги исследования будут использованы для проектирования корректирующих средств с иелью устранения нарушений осанки.

Ключевые слова: осанка, антропоморфологическое исследование, детское население, сколиоз. 


\begin{tabular}{|c|c|c|c|c|c|c|}
\hline \multirow{4}{*}{ Impact Factor: } & ISRA (India) & $=3.117$ & SIS (USA) & $=0.912$ & ICV (Poland) & $=6.630$ \\
\hline & ISI (Dubai, UAE & $=0.829$ & РИНЦ (Russia & $=0.156$ & PIF (India) & $=1.940$ \\
\hline & GIF (Australia) & $=0.564$ & ESJI (KZ) & $=8.716$ & IBI (India) & $=4.260$ \\
\hline & JIF & $=1.500$ & SJIF (Morocce & $=5.667$ & OAJI (USA) & $=0.350$ \\
\hline
\end{tabular}

\section{Введение}

Как известно, в мире активно происходит процесс акселерации населения, что особое влияние оказывает на физическое развитие детского организма. Процесс развития детского организма отличается от развития взрослого человека по ряду анатомо-физиологических особенностей $[1,2]$. Во время роста ребенка происходят изменения телосложения, пропорций организма, что часто приводит к нарушениям осанки. Кроме того, что хорошая осанка создает приятное впечатление на окружающих, она является источником здоровья.

Причины нарушения осанки детей могут быть как врожденными, так и приобретёнными. Также выделяют такие социальные факторы, как:

- Малоподвижный образ жизни;

- Неудобная мебель, несоответствующая росту ребенка;

- Недостаточное освещение рабочего стола;

- Ношение рюкзака на одном плече, либо использование сумки или пакета.

При всех вышеперечисленных ситуациях происходит фиксация неправильного положения тела, а через определенный период времени это переходит в привычку.

Неправильная осанка у детей процессе формирования организма вызывает ряд нарушений, в часности нарушается нормальное кровообрашения, происходит ухудшение памяти, развивается быстрая утомляемость, появляются боли в области позвоночника, ребенку тяжело долго сидеть и т.д. К сожалению, родители большое внимание уделяют питанию и образованию детей, а о нарушении осанки в школьном возрасте они узнают от врача тогда, когда проблема уже сформирована.
По статистике установлено, что в мире на сегодняшний день у 80-85\% детей от 6 до 13 лет выявлены разные виды нарушений осанки [3]. Статистика настораживает и создает необходимость своевременного выявления проблемы и применения корректирующих средств для профилактики сколиоза [4].

Для решения вышесказанной проблемы имеет большое значение антропоморфологическое исследование детского населения Грузии, которое в нашей стране в последний раз проводилось в $80 x$ годах прошедшего столетия $[5,6,7,8]$. Тогда как периодичность проведения таких исследований составляет 10 лет. Это тот период, в течение которого меняются размерные признаки фигуры, что обусловлено процессом акселерации, социоэкономическим уровнем жизни и изменениями физического развития детей. Проблема актуальна и имеет государственную значимость [9]. На это указывает тот факт, что в ряде стран Европы регулярно выделяются средства для проведения масштабного антропометрического исследования населения.

Целью проведенных исследований было выявления типов нарушений осанки и определение их процентных показателей в разных возрастных группах детей в результате антроморфологических исследований детей в одном из регионов Грузии-Имерети. Результаты проведенных в Грузии антропоморфологических исследований показали, что количественный показатель нарушения осанки у детей разной возрастной группы составляет - 44\% для детей младшего школьного возраста, 34\% для детей старшего школьного возраста, $28 \%$ для подростков (рис. 1а, б, в) 


\begin{tabular}{llllll} 
& ISRA (India) $=\mathbf{3 . 1 1 7}$ & SIS (USA) & $=\mathbf{0 . 9 1 2}$ & ICV (Poland) & $=\mathbf{6 . 6 3 0}$ \\
Impact Factor: & ISI (Dubai, UAE) $=\mathbf{0 . 8 2 9}$ & PUHL (Russia) $=\mathbf{0 . 1 5 6}$ & PIF (India) & $=\mathbf{1 . 9 4 0}$ \\
& GIF (Australia) $=\mathbf{0 . 5 6 4}$ & ESJI (KZ) & $=\mathbf{8 . 7 1 6}$ & IBI (India) & $=\mathbf{4 . 2 6 0}$ \\
& JIF & $\mathbf{1 . 5 0 0}$ & SJIF (Morocco) $=\mathbf{5 . 6 6 7}$ & OAJI (USA) & $\mathbf{0 . 3 5 0}$ \\
\hline
\end{tabular}

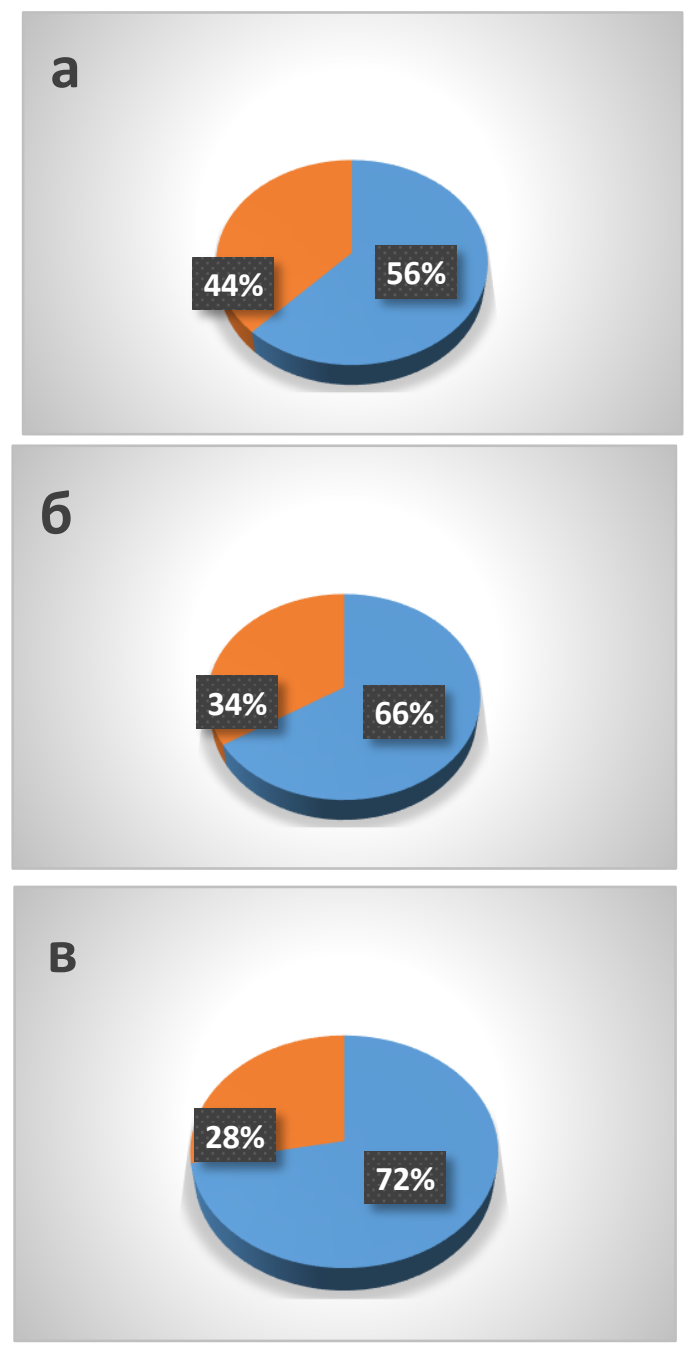

Рисунок 1 - Количественные показатели нарушения осанки: а - дети младшего школьного возраста, б - дети старшего школьного возраста, в - подростки

Во время исследования рассматривались 3 типа нарушений осанки у детей: сутулая спина, неровность плеч и выпрямленная спина. Результаты исследования (рис. 2,3,4) показали, что среди типов нарушения осанки наиболее часто встречалась «Сутулая спина», количественный показатель которой распределился по возрастным группам следующим образом: у детей младшего школьного возраста - 21\%, старшеклассников $21 \%$ и у подростков $14 \%$. 


\begin{tabular}{llllll} 
& ISRA (India) $=\mathbf{3 . 1 1 7}$ & SIS (USA) & $=\mathbf{0 . 9 1 2}$ & ICV (Poland) & $=\mathbf{6 . 6 3 0}$ \\
Impact Factor: & ISI (Dubai, UAE) $=\mathbf{0 . 8 2 9}$ & PUHL (Russia) $=\mathbf{0 . 1 5 6}$ & PIF (India) & $=\mathbf{1 . 9 4 0}$ \\
& GIF (Australia) $=\mathbf{0 . 5 6 4}$ & ESJI (KZ) $=\mathbf{8 . 7 1 6}$ & IBI (India) & $=\mathbf{4 . 2 6 0}$ \\
& JIF & $\mathbf{1 . 5 0 0}$ & SJIF (Morocco) $=\mathbf{5 . 6 6 7}$ & OAJI (USA) & $\mathbf{0 . 3 5 0}$ \\
\hline
\end{tabular}

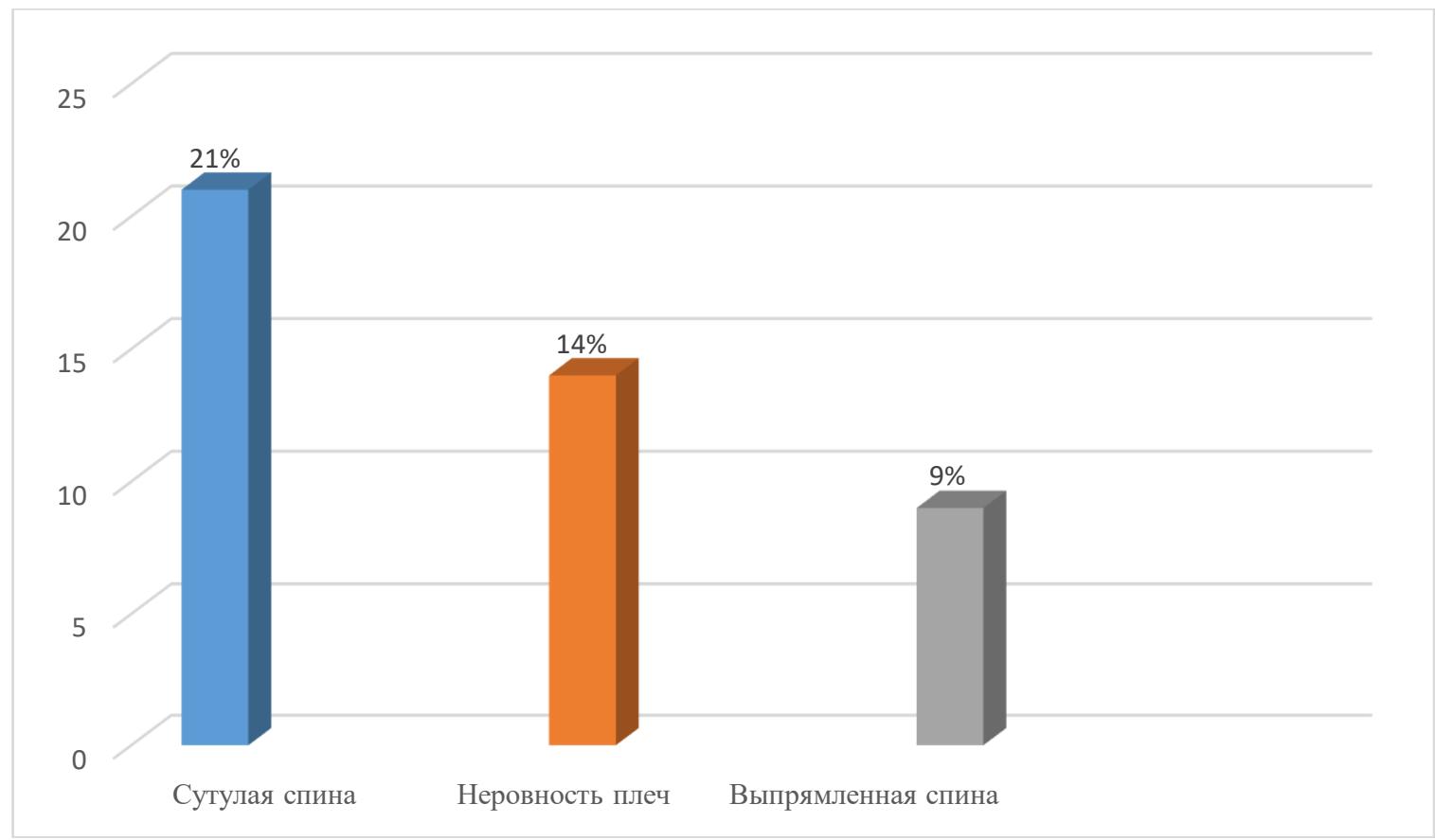

Рисунок 2 - Итоги определения типов нарушения осанки у детей младшего школьного возраста 25

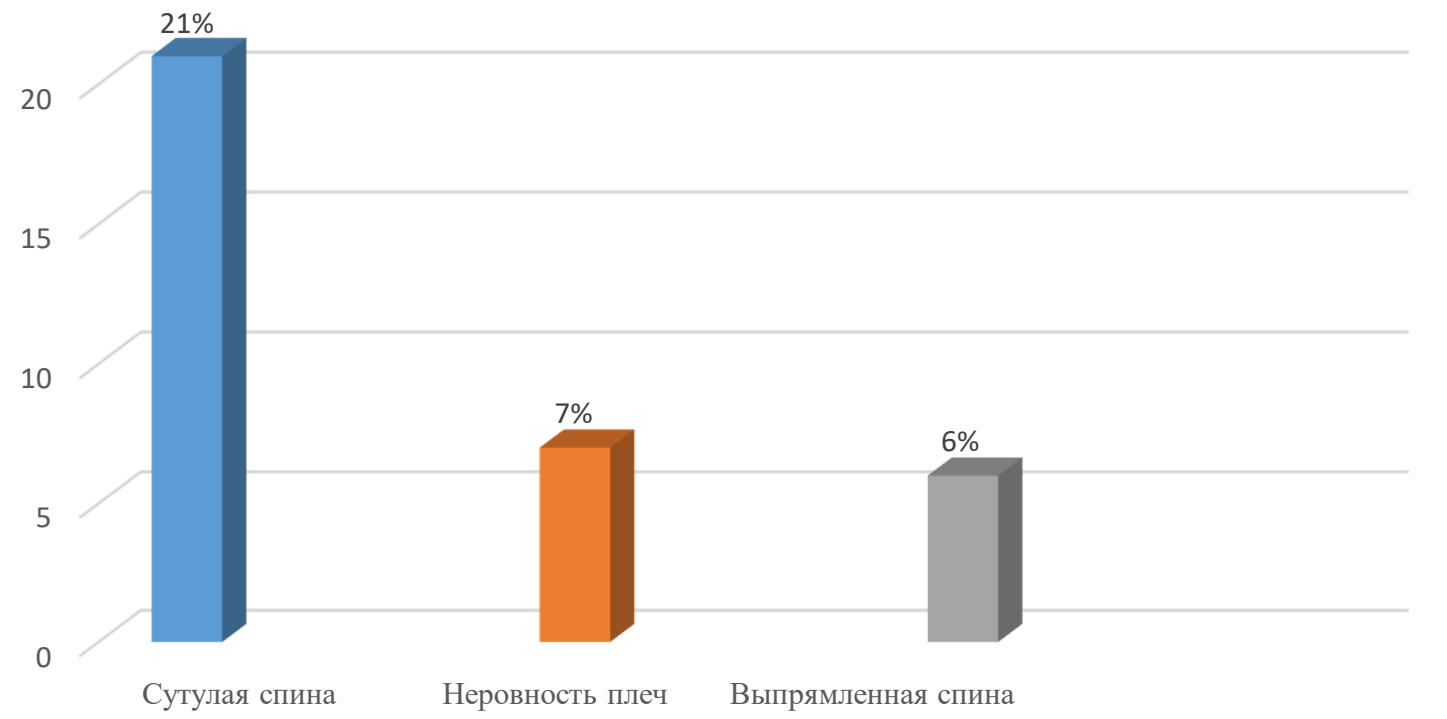

Рисунок 3 - Итоги определения типов нарушения осанки у детей старшего школьного возраста 


\begin{tabular}{|c|c|c|c|c|c|c|}
\hline \multirow{4}{*}{ Impact Factor: } & ISRA (India) & $=3.117$ & SIS (USA) & $=0.912$ & ICV (Poland) & $=6.630$ \\
\hline & ISI (Dubai, UAE & $=0.829$ & РИНЦ (Russia) & $=0.156$ & PIF (India) & $=1.940$ \\
\hline & GIF (Australia) & $=0.564$ & ESJI (KZ) & $=8.716$ & IBI (India) & $=4.260$ \\
\hline & JIF & $=1.500$ & SJIF (Morocco) & $=5.667$ & OAJI (USA) & $=0.350$ \\
\hline
\end{tabular}

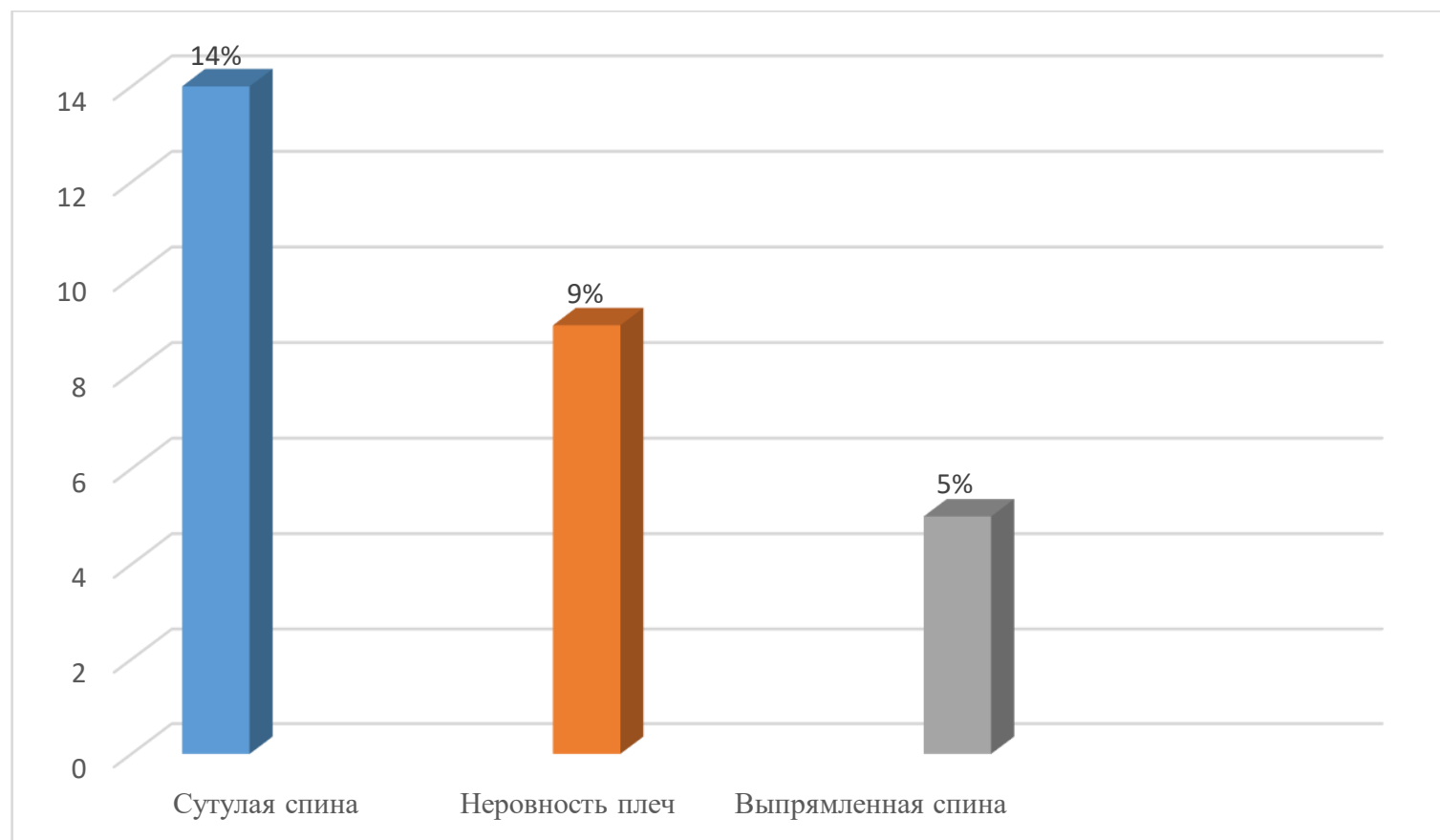

Рисунок 4 - Итоги определения типов нарушения осанки у детей подросткового возраста

Проведенные антропометрические исследования детей в регионе Имерети показали, что особенно часто встречаются случаи нарушения осанки у детей в возрасте от 6 до 13 лет. Таким образом выявилась необходимость проведения массовых антропоморфологических исследований детей по всей Грузии для своевременного выявления и устранения проблемы путем проведения соответствующих мероприятий.

Как известно, одной из множества причин нарушения осанки является одежда, несоответствующая по форме и размеру тело ребенка. Такая одежда может вызвать замедление процесса роста, нарушение функции внутренних органов и изменения осанки- отклонение от нормального состояния. На сегодняшний день в Грузии детское население снабжается одеждой иностранного производства, которая не предусматривает особенности антропометрических данных детского населения Грузии. Одежда не соответствует размеру и пропорциям детских фигур. К тому же как известно, в мире происходит ярко выраженный процесс акселерации детского населения, который вносит значительные изменения в размерной типологии [10]. Этот вопрос особенно проблематичен в Грузии, так как антропометрические исследования не проводились почти 40 лет, а существующие стандарты уже устаревшие.

Для устранения нарушения осанки детей большое значение имеют корректирующие средства. Существующие модели имеют ряд недостатков. Они не предусматривают антропометрических и морфологических данных детей конкретного региона, в нашем случае Грузии.

\section{Заключение}

Из вышесказанного следует заключить, что вопрос весьма актуален. При проведении массовых антропоморфологических исследований детского населения Грузии будет возможность установить случаи нарушений осанки, определить их виды и количественные показатели, что имеет большое значение для предотвращение дальнейших осложнений и профилактики сколиоза, что в свою очередь послужит основой для разработки корректирующих средств осанки и размерной типологии детей для отдельных половозрастных групп.

\section{References:}




\begin{tabular}{llllll} 
& ISRA (India) $=\mathbf{3 . 1 1 7}$ & SIS (USA) $=\mathbf{0 . 9 1 2}$ & ICV (Poland) & $\mathbf{= 6 . 6 3 0}$ \\
Impact Factor: & ISI (Dubai, UAE) $=\mathbf{0 . 8 2 9}$ & PUHЦ (Russia) $=\mathbf{0 . 1 5 6}$ & PIF (India) & $=\mathbf{1 . 9 4 0}$ \\
& GIF (Australia) $=\mathbf{0 . 5 6 4}$ & ESJI (KZ) & $\mathbf{8 . 7 1 6}$ & IBI (India) & $=\mathbf{4 . 2 6 0}$ \\
& JIF & $\mathbf{1 . 5 0 0}$ & SJIF (Morocco) $=\mathbf{5 . 6 6 7}$ & OAJI (USA) & $\mathbf{0 . 3 5 0}$ \\
\hline
\end{tabular}

1. Godina, E. Z. (2001). Dinamika processov rosta $i$ razvitija cheloveka. (p.346). Moscow: Medicina.

2. Zadorozhnaja, L.V. (1990). Vlijanie nekotoryh faktorov okruzhajushhej sredy na formirovanie osobennostej somaticheskogo razvitija detej $i$ podrostkov. (p.184). Vopr. antropologii.

3. (n.d.). Narushenie osanki. Retrieved 2019, from http://www.life-ergo.ru/information/articlesand-materials/advantage-forhealth/posture/postureflu

4. Krasikova, I. S. (2008). Vospitanie pravil'noj osanki. Lechenie narushenij osanki. (p.176). Saint-Petersburg: Korona-Vek.

5. (2001). Razmernaja tipologija naselenija $s$ osnovami anatomii i morfologii. Pod redakciej E.B. Kobljakovoj (Eds.). (p.286). Moscow.
6. Homutov, A. (2006). Antropologija. Moscow.

7. Kostin, J. A. (2005). Osnovy prikladnoj antropologii $i$ biomehaniki. uchebnoe posobie. (p.104). Ivanovo: IGTA.

8. Lysov, P. K., Nikitjuk, B. D., \& Sapin, M. R. (2003). Anatomija (s osnovami sportivnoj morfologii). Moscow: Medicina.

9. Dolidze, N. A., \& Chirgadze, K. A. (2018). Issledovanie teloslozhenij detskogo naselenija Gruzij. Materialy mezhdunarodnoj konferencij «Sovremennaja nauka i inovacionnaja praktika». Kutaisi.

10. Dunaevskaja, T. N., Kobljakova, E. B., \& Ivleva, G. S. (2002). Razmernaja tipologija naselenija s osnovami anatomii i morfologii. - 3-e izd., pererab. (p.216). Moscow. Legkaja industrija. 


\begin{tabular}{|c|c|c|c|c|c|c|}
\hline \multirow{4}{*}{ Impact Factor: } & ISRA (India) & $=3.117$ & SIS (USA) & $=0.912$ & ICV (Poland) & $=6.630$ \\
\hline & ISI (Dubai, UAE & $=0.829$ & РИНЦ (Russia) & $=0.156$ & PIF (India) & $=1.940$ \\
\hline & GIF (Australia) & $=0.564$ & ESJI (KZ) & $=8.716$ & IBI (India) & $=4.260$ \\
\hline & JIF & $=1.500$ & SJIF (Morocco) & $=5.667$ & OAJI (USA) & $=0.350$ \\
\hline
\end{tabular}

